\title{
Mapping theme trends and knowledge structure of labor analgesia: a quantitative, co-word biclustering analysis of data in 2000-2020
}

\author{
Xue Bai ${ }^{1}$, Zi-Xuan Song ${ }^{2}$, Yu-Xiao Wan ${ }^{3}$, Zhi-Qiang Feng ${ }^{4}$, Dong-Hai Yu ${ }^{5}$, \\ Dan-Dan Zhang ${ }^{2, *}$
}

\author{
${ }^{1}$ Shengjing Hospital of China Medical \\ University, Department of Health \\ Management, Shenyang, P. R. China \\ ${ }^{2}$ Shengjing Hospital of China Medical \\ University, Department of Obstetrics \\ and Gynecology, Shenyang, P. R. China \\ ${ }^{3}$ Shengjing Hospital of China Medical \\ University, Department of \\ Anesthesiology, Shenyang, P. R. China \\ ${ }^{4}$ Affiliated Zhongshan Hospital of Dalian \\ University, Department of \\ Anesthesiology, Dalian, P. R. China \\ ${ }^{5}$ Dalian Central Hospital, Department of \\ Anesthesiology, Dalian, P. R. China
}

\section{*Correspondence}

zhangdd@sj-hospital.org

(Dan-Dan Zhang)

\begin{abstract}
Background: The distribution knowledge structure and pattern of the literature on labor analgesia in PubMed were examined.

Methods: Scientific papers on labor analgesia published from 1 January, 2000 to 31 June, 2020 were retrieved. The extracted MeSH items were quantitatively analyzed by the Bibliographic Item Co-Occurrence Matrix Builder (BICOMB), and the high frequency $\mathrm{MeSH}$ items were identified. In gCLUTO software, repeated bisection method was used to Mountain visualisation, and the visual matrix was established. By constructing high-frequency MeSH terms co-occurrence matrix, strategic diagram and social network are further completed.

Results: The search strategy yielded 2870 papers, and the number of papers published annually had changed slightly during the study period. Among all extracted MeSH terms, 42 high-frequency MeSH terms were identified by consensus, and were divided into six categories by diclustering analysis. In the strategic diagram, the methods of labor analgesia, drug doses, and routes of administration were properly presented. In contrast, statistical and numerical data on obstetric analgesia were relatively underdeveloped, and management of pain during labor was undeveloped. In the social network analysis, the position status of each component was determined by the centrality values.

Conclusions: The findings on labor analgesia are relatively divergent, and the six research categories outlined in this study reflect the publication trends in the field of labor analgesia to some extent. Our quantitative bibliometric research across a 20-year span depicts the overall direction of the latest topics and provides some hints for researchers when launching new projects.
\end{abstract}

\section{Keywords}

Labor analgesia; Bibliometric analysis; Co-word analysis; Social network analysis

\section{Background}

The American College of Obstetrics and Gynecology (ACOG) pointed out that it was inhumane and unhealthy for women to suffer during childbirth $[1,2]$. The intense pain of childbirth can activate the sympathetic nervous system, increase the secretion of catecholamines, which cause a decrease in uterine blood flow and an increase in cardiac output and blood pressure [3]. Hyperventilation during contractions may cause respiratory alkalosis and left shift of oxygenated hemoglobin curve, reducing the oxygen supply to the fetus. In addition, fear and anxiety can aggravate labor pain, leading to an increase in the rate of cesarean section [4]. Women should be helped to give birth with smiles and joy instead of moaning, painful and struggling. The purpose of labor analgesia is to reduce labor pain and anxiety, and improve labor comfort and satisfaction $[5,6]$. However, there are so many choices for labor analgesia, and the impact of labor analgesia on the safety of mothers and babies, on labor process, contractions, intrapartum fever and postpartum depression are still controversial [5]. According to the bibliometric analysis method, our studies use cluster visualization analysis to show the thematic structure of childbirth analgesia; the strategic coordinates to show the importance and characteristics of each research topic in the overall disciplinary structure; the social network map to show the internal relations of the topics. The study summarizes the research hotspots and development trends of labor analgesia in the past 20 years, so as to facilitate researchers in this discipline to understand the frontiers of the discipline, further clarify their research goals, rationally plan the discipline layout, adjust the research direction, and concentrate limited strength in key areas. 


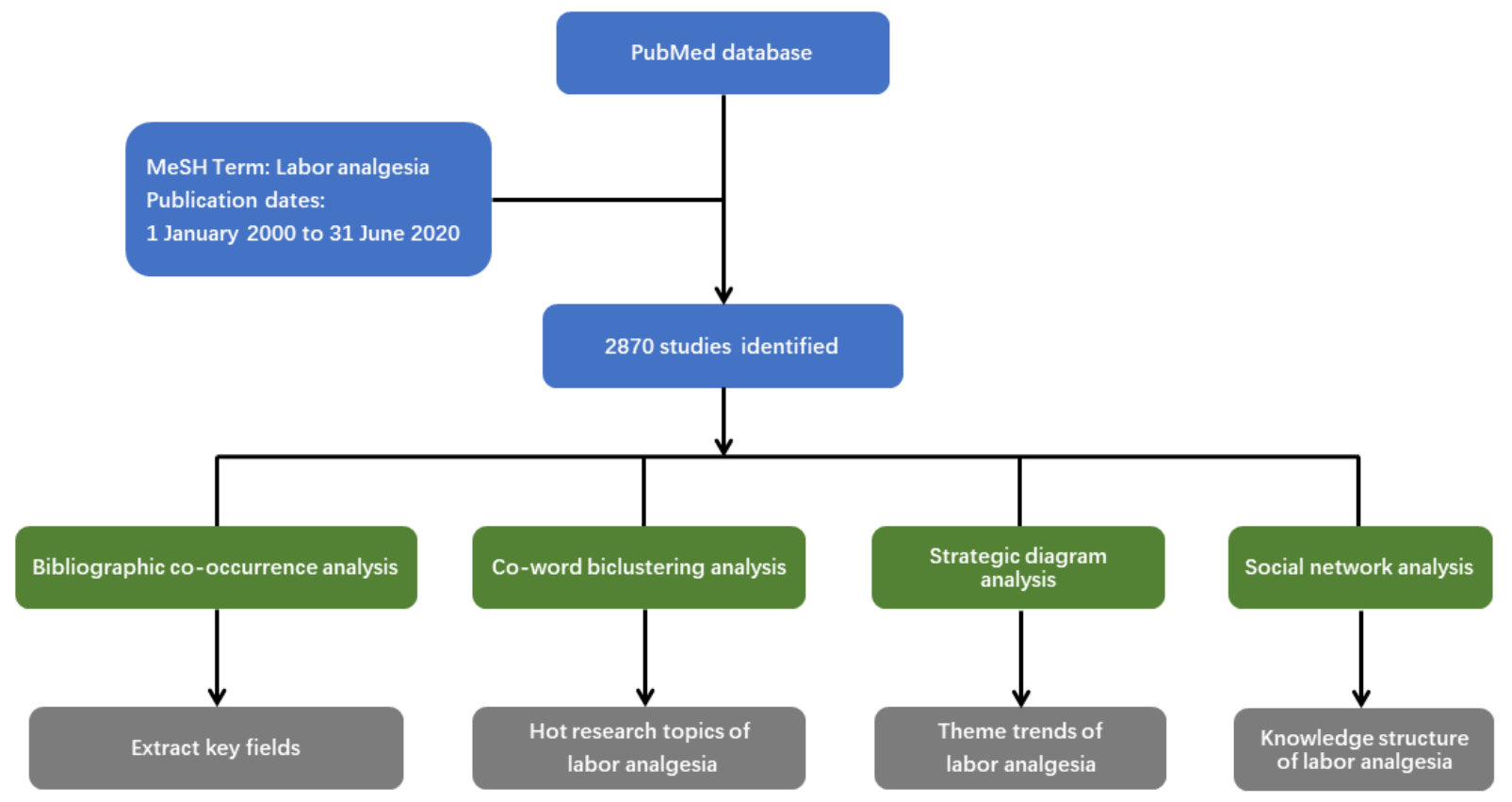

F I G U RE 1. The flowchart of literature selection.

\section{Data and methods}

\subsection{Data resource and search strategy}

In PubMed database of the US National Center for Biotechnology Information, Medical Subject Heading (MeSH) is used for indexing, cataloging, and searching for biomedical and healthrelated information which created by the National Library of Medicine. We retrieved and downloaded the data by using $\mathrm{MeSH}$ term as the retrieval category. The retrieval term was set as "Labor analgesia" [MeSH]. A total of 2870 articles published between 1 January 2000 and 31 June 2020 were retrieved. The data were saved in XML format including title, author, country, MeSH terms, and publication year of each study. The specific flowchart is shown in Fig. 1.

\subsection{Data extraction and bibliographic matrix building}

The required data were extracted from the PubMed database. Two investigators (Zhang and Bai) independently conducted the primary search by screening the full texts, titles and, in some cases, abstracts, of the studies. The agreement rate between them was 0.90, which indicated strong agreement [7]. Differences, if any, were discussed. The co-occurring matrices and term-source article relationships were checked using the Bibliographic Item Co-Occurrence Matrix Builder (BICOMB) software developed by Professor Cui of the China Medical University that is available freely online [8]. BICOMB was utilized to analyze the contribution characteristics of country, journal, year of publication, and main/subheadings MeSH terms. In addition, the numbers of high-frequency main $\mathrm{MeSH}$ terms/subheadings were defined by the $\mathrm{H}$ index.

\subsection{MeSH terms Biclustering analysis}

Biclustering analysis was performed on high-frequency main $\mathrm{MeSH}$ terms/subheadings and PubMed Unique Identifiers of the labor analgesia-related studies. The main MeSH terms/subtitles were classified according to the term-source article matrix. Repeated bisection method is used in gCLUTO software to visualize mountain body and establish visualization matrix. The peaks in the three-dimensional (3D) terrain were marked by numbers, representing the clusters analysed by biclustering. The data of the associated clusters are reflected by the colour, height, volume and position of the peaks, and the relative position of each peak is the most informative attribute in the figure. The height of each peak represents the internal resemblance of a cluster, the distance between peaks reflects the correlation between clusters and the volume of the peak is proportional to the number of main $\mathrm{MeSH}$ terms/subheadings. In addition, the peak colour is the internal standard deviation of the objects in the cluster, and blue is high deviation, red is low. Based on the results of the biclustering analysis, the structures related to the research focus areas were further analysed.

\subsection{Strategic diagram analysis}

Taking theme centrality and density as axes, we constructed a two-dimensional strategy diagram. The horizontal axis represented the centrality, and the vertical axis represented the density. The Centrality referred to the intensity of interaction between domains, and the density referred to the intensity of internal connection in a certain field. The coordinates were divided into four quadrants by two axes, and we used strategic diagram calculation formula to distribute the results of the subject words main MeSH terms/subheadings to four quadrants. 
A

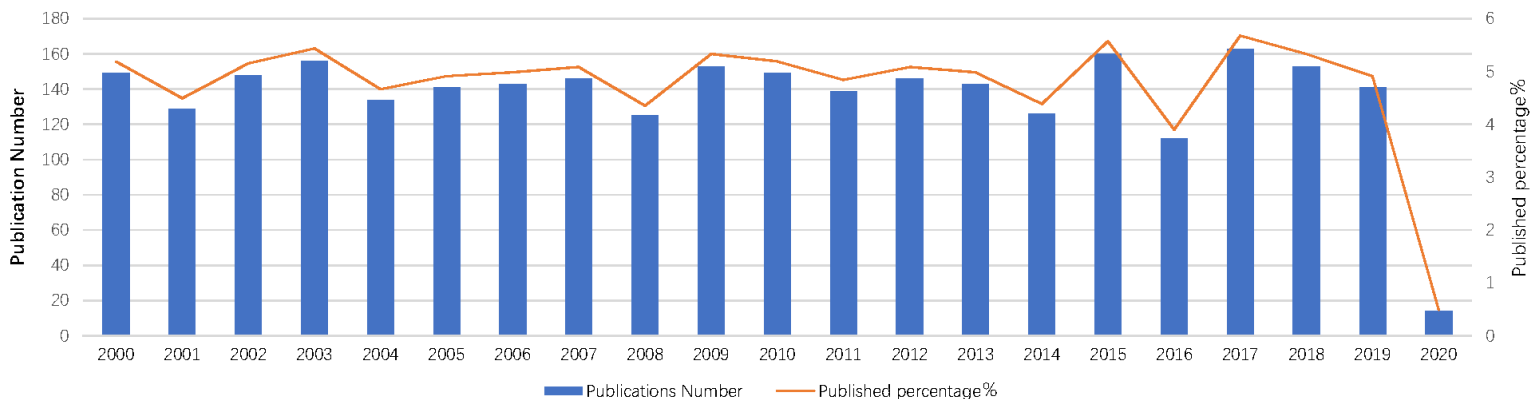

B

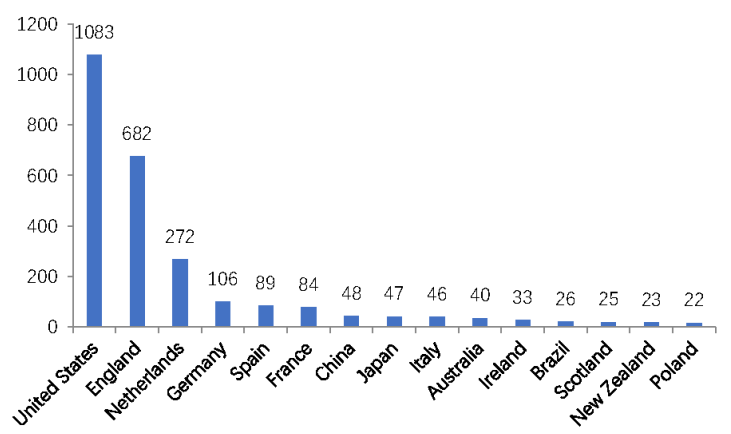

C

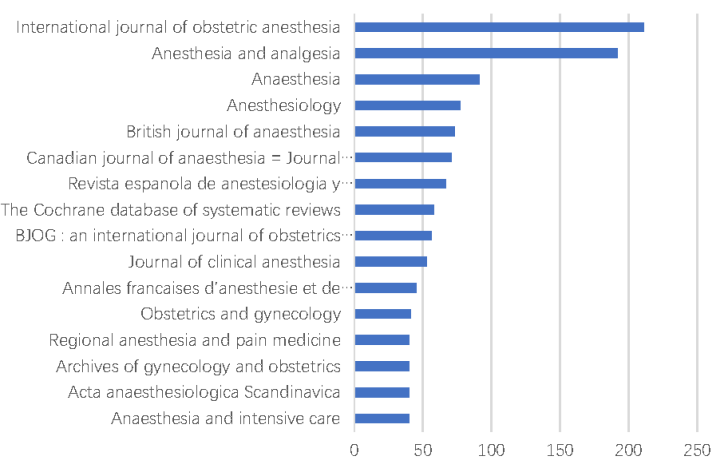

F I G URE 2. Characteristics of labor analgesia-related publications. Publication years (A), publication contrary (B) and high frequently journals $(\mathrm{C})$.

\subsection{Social network analysis}

Ucinet 6.0 software was used to draw the social network analysis network by analyzing the co-occurrence matrix of high-frequency main MeSH terms/subheadings. Then social network analysis revealed the intrinsic link of labor analgesia. With NetDraw 2.084 software, the main MeSH terms/subheadings was to be submitted and visualised into a two-dimensional network. In the network, each node represented a main MeSH terms/subheadings, and each link represented co-occurrence frequency. The tightness of the network was respected by calculating the keyword density. We measured three centralities of MeSH terms/subheadings (closeness, betweenness, and degree) to compare the positions of different nodes in the structure of labor analgesia network.

\section{Results}

The search strategy yielded a total of 2870 publications that were included in this study. The data on labor analgesiarelated publications in the designated study period are shown in Fig. 2-A. The highest number of articles on labor analgesia was published in the United States, accounting for $37.73 \%$ of all the retrieved studies, followed by England and the Netherlands (Fig. 2-B). The top ranking 15 journals are shown in Fig. 2-C. The International Journal of Obstetric Anesthesia published the highest number of labor Indonesia-related articles (211, 7.34\%), followed by Anesthesia and Analgesia (192, 6.68\%) and Anesthesia (91, 3.16\%).

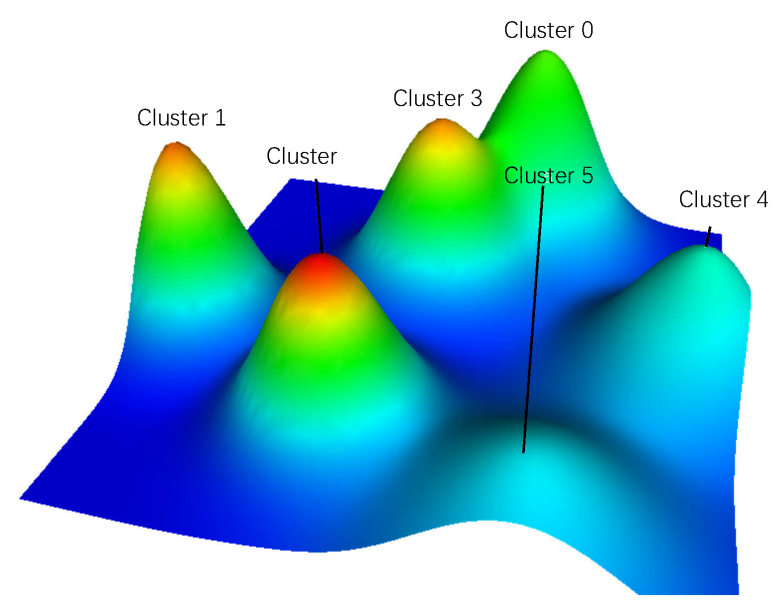

F I G U RE 3. A visualized mountain map biclustering of major MeSH terms with high frequency and articles on Labor Analgesia.

\subsection{Research hot spots according to MeSH term clusters}

Among the labor analgesia-related publications, highfrequency main MeSH terms/subheadings had a cumulative frequency of $43.40 \%$ and were the hot research topics over the past 20 years. The high-frequency main $\mathrm{MeSH}$ terms/subheadings were analysed, and six clusters were identified by biclustering analysis. Accordingly, 41 highfrequency main $\mathrm{MeSH}$ terms/subheadings were classified into six clusters (Fig. 3). The hierarchical trees on the left and top sides represent the high-frequency main $\mathrm{MeSH}$ terms/subheadings and PubMed Unique Identifier (PMID), respectively. Furthermore, the themes in the representative 
TA B L E 1. Highly Frequent Terms in Labor Analgesia.

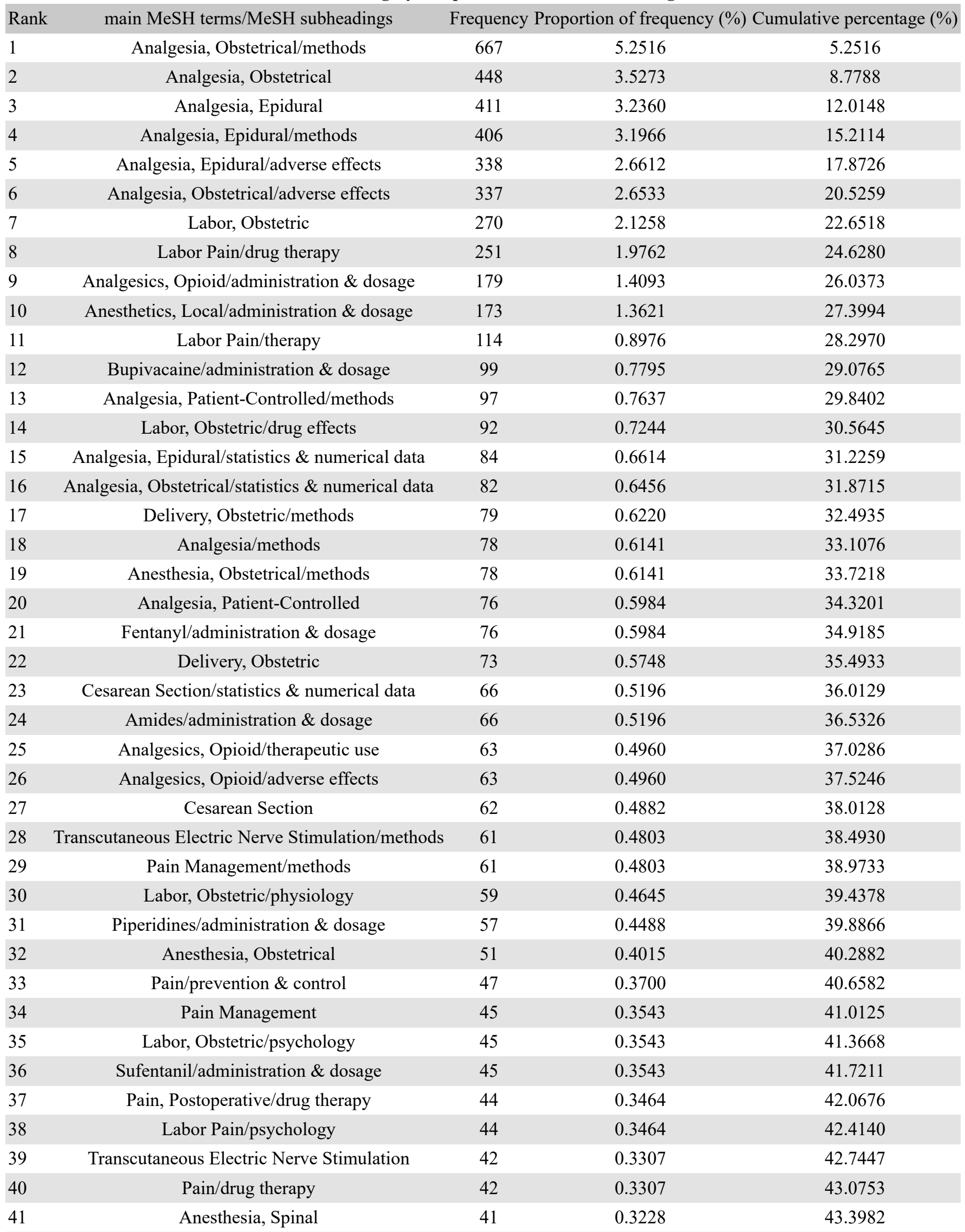

papers in each cluster were summarised. Considering the hot research topics based on MeSH term clusters, the cumulative frequency of the 41 high-frequency main $\mathrm{MeSH}$ terms/subheadings was $43.3982 \%$ (Table 1). 


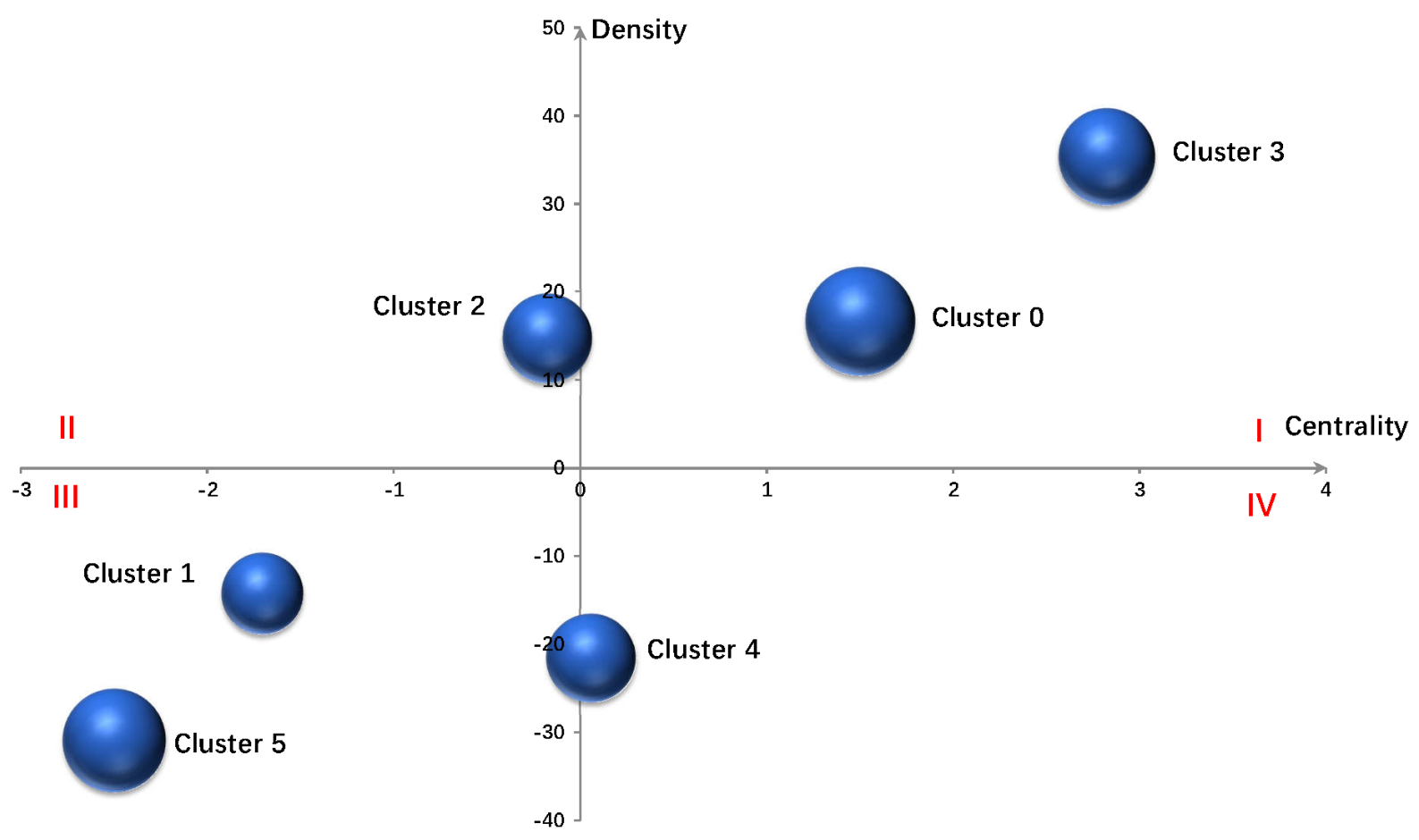

F I G U RE 4. Strategic diagram for Labor Analgesia. Cluster No. 0,3 locate in Quadrant I, representing that researches on analgesia methods, administration and dosage are in the core status with high density and centrality. Cluster No. 2 locate in Quadrant II, representing that researches on labor analgesia related to adverse effects are in the peripheral and developed status. Cluster No. 1, 5 locate in Quadrant III, indicating that researches on statistics and numerical data, as well as psychology related studies on labor analgesia are not mature at the edge of the research field. Cluster No. 4 locate in Quadrant IV, indicating that researches on pain management are in the central and undeveloped status.

\subsection{Strategic diagram for labor analgesia}

Quadrant I (upper-right) represents motor themes with strong centrality and high density. Quadrant II (upper-left) represents specialised themes with high density, but inadequate external interactions. Quadrant III (lower-left) contains themes with low density and inadequate centrality, and these themes may be marginal topics. Quadrant IV (lower-right) contains themes with strong centrality but lacking internal maturation. In the strategic diagrams, the themes are represented by spheres of different sizes, which are organised in different quadrants according to their internal and external cohesion (density and centrality).

As shown in Fig. 4, the area of the spheres is proportional to the number of high-frequency main MeSH terms/subheadings. Cluster No. 0 and 3 are located in Quadrant I, indicating that studies on analgesia methods, administration, and dosage are in the core position with high density and centrality. Cluster No. 2 is located in Quadrant II, indicating that research on labor analgesia related to adverse effects is in the peripheral and developed position. Cluster No. 1 and 5 are located in Quadrant III, indicating that statistical, numerical, and psychologyrelated studies on labor analgesia are not mature and at the edge of the research field. Cluster No.4 is located in Quadrant IV, indicating that research on pain management is in the central and undeveloped position. The strategic diagram shows the development and trends in each cluster over the past 20 years.

\subsection{Social network analysis of labor analgesia}

The density value of labor analgesia-related studies was 0.6244 . This indicates that the overall structure of the network is relatively close. Degree, closeness, and betweenness were used as centrality parameters to build the social network analysis network (Fig. 5 and Table 2).

In the network of labor analgesia studies published in the last 20 years, 20 main MeSH terms/subheadings had a high degree of centrality, including the top 15 high-frequency ones. "Analgesia, Obstetrical/methods" had the highest degree of centrality and betweenness centrality. It played the most significant mediating role in the network. "Analgesia, Epidural" and "Analgesia, Obstetrical" also had high betweenness centrality, indicative of their prominent mediating roles in the network. The mean betweenness centrality was $7.512 \pm 7.396$ (Table 3).

\section{Discussion}

MeSH terms can reveal the content of studies, research status, and trends in different disciplines. According to the analytical results obtained from the BICOMB software, the distribution characteristics of the "Labor Analgesia" [MeSH] literature in the last 20 years maintained a horizontal trend, with a slight decrease in 2016. Our analysis showed that the United States and the United Kingdom were the largest contributors, which can be explained by the fact that English is the first language 


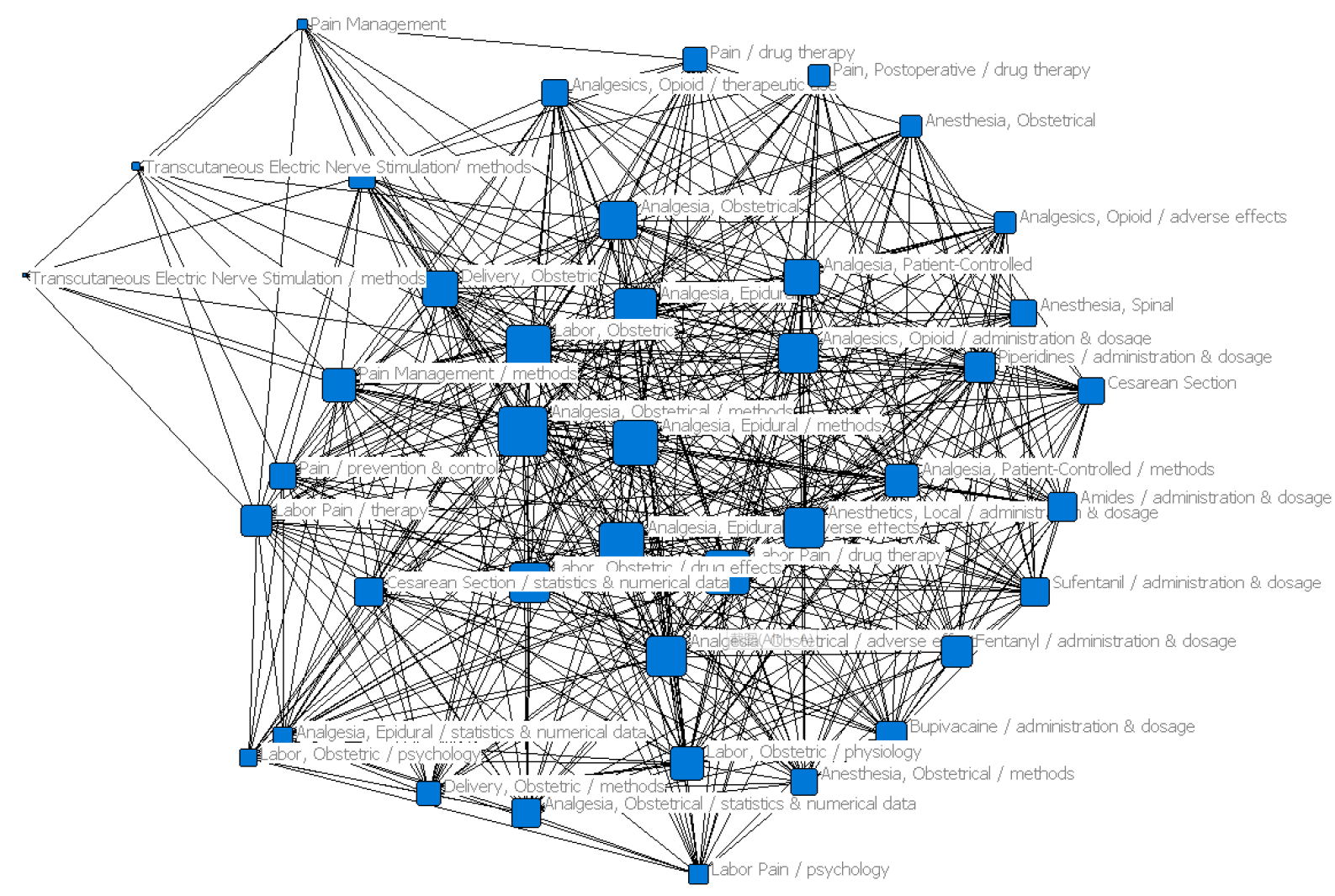

\section{F I G U R E 5. Labor Analgesia of 42 high-frequency MeSH terms.}

of these two countries. To systematically examine the basic research direction of labor analgesia, we performed lexical meaning analysis. By co-word analysis, closely related MeSH terms were gathered into clusters.

Cluster 3 involved studies on the methods of labor analgesia. There are many methods of providing labor analgesia, among which drug analgesia mainly includes inhalation anaesthesia, intravenous anaesthesia, and spinal anaesthesia, and non-drug analgesia mainly includes Lamaze respiratory analgesia, doula delivery and water delivery. At present, mainstream research focuses on the methods of drug analgesia. Previous studies reported that inhaled analgesia was simple to use and effective, but excessive inhaled anaesthetics can have the effects of general anaesthesia, inhibit protective reflex, and increase the risk of aspiration [9]. The second focus is on intravenous analgesia, including fentanyl, remifentanil and tramadol. In recent years, remifentanil has shown a significant advantage in labor analgesia which has high lipid solubility and is fast acting. When there are contraindications to intraspinal anaesthesia and it is difficult to tolerate labor pain, intravenous labor analgesia can be considered $[10,11]$. However, risks such as respiratory depression, excessive sedation, and decreased blood oxygen saturation, require close monitoring of maternal vital signs and foetal heart conditions [12]. At present, the most effective method of labor analgesia is that of injecting a small dose of anaesthetics into the maternal body through the spinal canal [13], mainly continuous infusion epidural analgesia (CIEA), patient-controlled epidural analgesia (PCEA), combined spinal epidural analgesia (CSEA), and walkable epidural analgesia (WEA). At present, PCEA is the most widely used method.
It has a good analgesic effect and can be administered by the pregnant women themselves, satisfying the needs of pregnant women for different pain degrees. Bupivacaine or ropivacaine combined with fentanyl or sufentanil are commonly used as spinal canal injections. Ropivacaine, as an amide local anaesthetic, rarely crosses the placental barrier, and studies have shown that when ropivacaine is used in combination with sufentanil, the dosage of local anaesthetic can be reduced and the onset time can be shortened [14].

Cluster 0 consists of studies on drug doses and routes of administration. Epidural labor analgesia is still dominated by low-concentration local anaesthetics and opioids, known as "walkable labor analgesia". The analgesic durations of ropivacaine-sufentanil and levobupivacaine-sufentanil are significantly longer than that of bupivacaine-sufentanil, and ropivacaine also shows unique "sensorimotor separation", which significantly reduces the incidence of motor blockade $[15,16]$. When combined with $2 \mathrm{~g} / \mathrm{mL}$ fentanyl, $0.1 \%$ ropivacaine was associated with a lower incidence of motor block than $0.1 \%$ bupivacaine. However, there were no significant differences in the pain score, device-assisted birth rate, caesarean section rate, incidence of Apgar score $<7$, maternal satisfaction, time of first labor, time of second labor, oxytocin use, analgesic onset time, and analgesic duration [17]. Moreover, epidural with a low concentration of local anaesthetic $(\leq 0.1 \%$ bupivacaine or $\leq 0.17 \%$ ropivacaine) reduced the degree of motion block, increased autonomous activity, decreased urinary retention, shortened second labor, and did not lead to changes in the pain score $[18,19]$. Technically, the CIEA + PCEA group had a reduced number of rescue doses compared with 
TA B L E 2. Individual centrality of Labor Analgesia research.

\begin{tabular}{|c|c|c|c|c|}
\hline Rank & main MeSH terms/subheadings & Degree & Betweenness & Closeness \\
\hline 1 & Analgesia, Obstetrical/methods & 39 & 34.416 & 41 \\
\hline 2 & Labor, Obstetric & 36 & 28.287 & 44 \\
\hline 3 & Labor Pain/drug therapy & 35 & 12.818 & 45 \\
\hline 4 & Analgesia, Epidural/methods & 35 & 17.379 & 45 \\
\hline 5 & Analgesia, Epidural/adverse effects & 35 & 13.426 & 45 \\
\hline 6 & Analgesia, Epidural & 34 & 19.052 & 46 \\
\hline 7 & Analgesia, Obstetrical/adverse effects & 33 & 10.737 & 47 \\
\hline 8 & Analgesics, Opioid/administration \& dosage & 32 & 7.815 & 48 \\
\hline 9 & Anesthetics, Local/administration \& dosage & 32 & 8.927 & 48 \\
\hline 10 & Labor, Obstetric/drug effects & 32 & 9.92 & 48 \\
\hline 11 & Analgesia, Obstetrical & 31 & 17.757 & 49 \\
\hline 12 & Analgesia, Patient-Controlled & 29 & 6.719 & 51 \\
\hline 13 & Delivery, Obstetric & 29 & 9.004 & 51 \\
\hline 14 & Analgesia, Patient-Controlled/methods & 28 & 6.764 & 52 \\
\hline 15 & Pain Management/methods & 28 & 14.318 & 52 \\
\hline 16 & Labor, Obstetric/physiology & 27 & 5.843 & 53 \\
\hline 17 & Labor Pain/therapy & 25 & 13.179 & 55 \\
\hline 18 & Fentanyl/administration $\&$ dosage & 25 & 3.104 & 55 \\
\hline 19 & Bupivacaine/administration \& dosage & 25 & 3.189 & 55 \\
\hline 20 & Piperidines/administration $\&$ dosage & 25 & 3.656 & 55 \\
\hline 21 & Cesarean Section/statistics \& numerical data & 24 & 3.569 & 56 \\
\hline 22 & Amides/administration \& dosage & 24 & 2.905 & 56 \\
\hline 23 & Sufentanil/administration \& dosage & 24 & 2.818 & 56 \\
\hline 24 & Analgesia, Obstetrical/statistics \& numerical data & 24 & 4.196 & 56 \\
\hline 25 & Pain/prevention \& control & 23 & 6.786 & 57 \\
\hline 26 & Anesthesia, Obstetrical/methods & 23 & 3.048 & 57 \\
\hline 27 & Cesarean Section & 22 & 2.51 & 58 \\
\hline 28 & Analgesia/methods & 22 & 10.076 & 58 \\
\hline 29 & Analgesics, Opioid/therapeutic use & 22 & 2.609 & 58 \\
\hline 30 & Anesthesia, Spinal & 22 & 2.728 & 58 \\
\hline 31 & Delivery, Obstetric/methods & 21 & 2.309 & 59 \\
\hline 32 & Pain/drug therapy & 21 & 4.079 & 59 \\
\hline 33 & Analgesics, Opioid/adverse effects & 20 & 1.306 & 60 \\
\hline 34 & Anesthesia, Obstetrical & 20 & 2.619 & 60 \\
\hline 35 & Pain, Postoperative/drug therapy & 19 & 2.281 & 61 \\
\hline 36 & Labor Pain/psychology & 18 & 2.643 & 62 \\
\hline 37 & Analgesia, Epidural/statistics \& numerical data & 18 & 1.788 & 62 \\
\hline 38 & Labor, Obstetric/psychology & 16 & 1.088 & 64 \\
\hline 39 & Pain Management & 11 & 1.81 & 69 \\
\hline 40 & Transcutaneous Electric Nerve Stimulation & 9 & 0.421 & 71 \\
\hline 41 & Transcutaneous Electric Nerve Stimulation/methods & 6 & 0.1 & 74 \\
\hline
\end{tabular}

PCEA alone, but increased instrument-assisted delivery rates and prolonged second leabor [20]. Recently, programmed intermittent epidural bolus (PIEB) has been used, which can be regularly administered by pulse. It not only brings analgesic drugs in full contact with the spinal nerve in the epidural cavity during each pulse administration but also provides the next 
TA B L E 3. Descriptive statistics for centrality measure about Labor Analgesia.

\begin{tabular}{lcccc} 
Centralization & MeanSD & Min & Max & Network centralization (\%) \\
Degree & $24.976 \pm 7.253$ & 6.000 & 39.000 & 36.86 \\
Betweenness & $7.512 \pm 7.396$ & 0.100 & 34.416 & 3.54 \\
Closeness & $55.024 \pm 7.253$ & 41.000 & 74.000 & 49.01 \\
\hline
\end{tabular}

pulse administration before the drug action from the last pulse disappears, providing a sustained and stable analgesic effect. PIEB significantly increased maternal satisfaction compared with CEI, and reduced the incidence of pain outbreak, movement block, instrument-assisted birth, analgesic drug dosage, and PCEA [21, 22]. Foreign authors Leo [23] and Patkar [24] et al. conducted comparative studies with PIEB and CEI groups and found that the analgesic effect in the two groups was similar. Leo [23] stated that the consumption of anaesthetic drugs per unit time in the PIEB group was also lower than that in the CEI group, and the onset time in the PIEB group was also relatively fast. In addition, computer-integrated patient-controlled epidural analgesia (CIPCEA) is an effective method of providing labor analgesia by automatically adjusting the injection rate according to the needs of the woman, thus reducing the incidence of pain outbreaks, the use of local anaesthetics, and the workload of the anaesthesiologist [25].

Cluster 2 is related to adverse reactions to labor analgesia. Most studies have not reported rare or severe adverse events associated with epidural labor analgesia [26]. Low-dose opioid analgesics combined with local anaesthetics are commonly used in spinal anaesthesia, but opioids often lead to a series of adverse reactions such as nausea, vomiting, itchy skin, fever, foetal tachycardia, urinary retention, neonatal respiratory depression, and others [24, 27]. ElKerdawy [28, 29] stated that epidural labor analgesia can cause postpartum hypotension in women with preeclampsia; Shanbin et al. [17] pointed out that in addition to the side effects caused by opioids, epidural labor analgesia also led to back pain (10\%) and chills (5\%) in women and bradycardia in new-borns $(10 \%)$. Second, intrapartum fever is also a common complication of labor analgesia. The causes of disease may be related to the changes in maternal body temperature regulation function, the increase in obstetric heat, the high ambient temperature in the delivery room, and the differences in maternal population and obstetric management approaches. Other studies have suggested that the main mechanism may be non-infectious inflammation, such as an increase in serum interleukin-6 level [22]. Of note, Pavithra et al. [30] pointed out that epidural analgesia is an invasive operation, and the incidence of headache $(87.0 \%$ vs. $8.7 \%, P$ $<0.001)$, low back pain (47.2\% vs. $19.6 \%, P=0.002)$, neck pain $(30.1 \%$ vs. $2.2 \%, P<0.001)$, hearing problems $(13.8 \%$ vs. $0, P=0.02)$, and visual symptoms $(19.5 \%$ vs. $0, P=$ 0.002 ) is higher in puerperas after a dural puncture accident than in normal puerperas. The data indicate that long-term complications of analgesia puncture require attention.

Clusters 1 and 5 represented statistical and numerical data on obstetric analgesia. Many studies have suggested that epidural analgesia is initiated when the cervix is opened to $\geq 3 \mathrm{~cm}$, and epidural analgesia is stopped when the cervix is fully opened. However, in these cases, the duration of epidural analgesia was short and satisfaction was low. Emerging maternal data are changing previous perceptions. Wong et al. [31] showed that, compared with intravenous analgesia, intra-spinal analgesia at early labor (cervix dilation $<4 \mathrm{~cm}$ ) did not increase the caesarean section rate, and the analgesia was effective, and the labor process was shortened. A randomised, controlled study involving 12,793 women [32] showed that epidural analgesia during the incubation period of labor (dilation of the cervix $\geq$ $1 \mathrm{~cm}$ ) did not prolong labor compared with active labor (cervix $\geq 4 \mathrm{~cm}$ ) nor did it increase the caesarean section rate. Other studies showed that discontinuing epidural analgesia close to the time of the opening of the cervix does not reduce the rate of instrumentally assisted delivery but increases the incidence of second-stage hypoanalgesia [33]. The effect of epidural labor analgesia on labor process is still controversial in Academia. In addition, differences among ethnicities, midwifery techniques, and medical expertise may have different effects on the interpretation and treatment of labor pain. Therefore, highquality, large-sample prospective randomised controlled trials are needed to confirm all above clinically [34].

Cluster 4 represents the management of pain during labor. It has been found that the expression of $\mu$ opioid receptor genes is related to pain sensitivity and can affect the analgesic effect of labor analgesia. A meta-analysis found that fentanyl ED50 was significantly lower in women with homozygous and heterozygous GJJ8 alleles than in women with homozygous wild-type A118 alleles who received epidural labor analgesia [35]. A similar study found that women with homozygous and heterozygous G118 alleles were 1.25 times more likely to receive sufentanil epidural analgesia than women with the wild-type A118 allele [36]. However, some studies have found that the presence or absence of the $G$ allele does not affect the analgesic effect of opioids during epidural delivery [37]. In addition, epidural injections of clonidine and neostigmine significantly prolonged analgesia and reduced the rate of hourly local anaesthetic and opioid use. There was no significant difference between the two drugs in total labor time, delivery mode (caesarean section or device-assisted delivery rate), and neonatal Apgar score, and no significant adverse reactions were found [38].

Amounts of studies has shown that epidural labor analgesia is safe and effective, and the incidence of maternal and infant adverse reactions is low [2]. At present, it is still the mainstream method of labor analgesia. Although this study focuses on research hotspots and future research trends of labor analgesia, it also has its own limitations. For example, in the data analysis, only high-frequency words are selected as the analysis object, and data with low frequency are ignored, which will lead to certain errors in the research results. In addition, the inclusion of more articles and the relaxation of the control of article quality may some limitations of this study. 
Further studies are needed to evaluate the effects of epidural analgesia on maternal and infant outcomes, especially longterm neurobehavioral effects on neonates.

\section{AUTHOR CONTRIBUTIONS}

Xue Bai and Dandan Zhang designed the study and drafted the manuscript; Yuxiao Wan searched strategy; Zhiqiang Feng and Donghai Yu designed the statistical analysis plan; Dandan Zhang reviewed the manuscript. All authors take responsibility for appropriate content.

\section{ACKNOWLEDGMENT}

We would like to express our gratitude to all those who helped us during the writing of this manuscript Thanks to Library of China Medical University for providing electronic data resources; thanks to all the peer reviewers for their opinions and suggestions.

\section{CONFLICT OF INTEREST}

The authors declare that they have no competing interests.

\section{REFERENCES}

[1] American College of Obstetricians and Gynecologists. Guidelines for Perinatal Care. American Academy of Pediatrics. 2002.

[2] Obstetric care consensus No. 2: levels of maternal care. Obstetrics \& Gynecology. 2015; 125: 502-515.

[3] Dexter F. Visual analog scale scores for labor pain. Anesthesia and Analgesia. 1999; 88: 1421.

[4] Ogboli-Nwasor E, Adaji S, Bature S, Shittu O. Pain relief in labor: a survey of awareness, attitude, and practice of health care providers in Zaria, Nigeria. Journal of Pain Research. 2011; 4: 227-232.

[5] El-Wahab N, Robinson N. Analgesia and anaesthesia in labour. Obstetrics, Gynaecology \& Reproductive Medicine. 2011; 21: 137-141.

[6] Gu D, Yang X, Deng S, Liang C, Wang X, Wu J, et al. Tracking knowledge evolution in cloud health care research: knowledge map and common word analysis. Journal of Medical Internet Research. 2020; 22: e15142.

[7] Landis JR, Koch GG. A one-way components of variance model for categorical data. Biometrics. 1977; 33: 671-679.

[8] Li F, Li M, Guan P, Ma S, Cui L. Mapping publication trends and identifying hot spots of research on Internet health information seeking behavior: a quantitative and co-word biclustering analysis. Journal of Medical Internet Research. 2015; 17: e81.

[9] Ling WX. Clinical observation on nitrogen monoxide for delivery analgesia. Chinese Medicine Modern Distance Education of China. 2013.

[10] Ismail MT, Hassanin MZ. Neuraxial analgesia versus intravenous remifentanil for pain relief in early labor in nulliparous women. Archives of Gynecology and Obstetrics. 2013; 286: 1375-1381.

[11] Tveit TO, Seiler S, Halvorsen A, Rosland JH. Labour analgesia: a randomised, controlled trial comparing intravenous remifentanil and epidural analgesia with ropivacaine and fentanyl. European Journal of Anaesthesiology. 2012; 29: 129-136.

[12] Hill JB, Alexander JM, Sharma SK, McIntire DD, Leveno KJ. A comparison of the effects of epidural and meperidine analgesia during labor on fetal heart rate. Obstetrics and Gynecology. 2003; 102: 333-337.

[13] Bucklin BA, Hawkins JL, Anderson JR, Ullrich FA. Obstetric anesthesia workforce survey: twenty-year update. Anesthesiology. 2005; 103: 645653.

[14] Shen X, Li Y, Xu S, Wang N, Fan S, Qin X, et al. Epidural analgesia during the second stage of labor: a randomized controlled trial. Obstetrics and Gynecology. 2017; 130: 1097-1103.
[15] Lv B, Wang W, Wang Z, Wang X, Wang J, Fang F, et al. Efficacy and safety of local anesthetics bupivacaine, ropivacaine and levobupivacaine in combination with sufentanil in epidural anesthesia for labor and delivery: a meta-analysis. Current Medical Research and Opinion. 2014; 30: 2279-2289.

[16] Li Y, Hu C, Fan Y, Wang H, Xu H. Epidural analgesia with amide local anesthetics, bupivacaine, and ropivacaine in combination with fentanyl for labor pain relief: a meta-analysis. Medical Science Monitor. 2015; 21: 921-928.

[17] Guo S, Li B, Gao C, Tian Y. Epidural analgesia with bupivacaine and fentanyl versus ropivacaine and fentanyl for pain relief in labor: a metaanalysis. Medicine. 2015; 94: e880.

[18] Sultan P, Murphy C, Halpern S, Carvalho B. The effect of low concentrations versus high concentrations of local anesthetics for labour analgesia on obstetric and anesthetic outcomes: a meta-analysis. Canadian Journal of Anesthesia. 2013; 60: 840-854.

[19] Craig MG, Grant EN, Tao W, McIntire DD, Leveno KJ. A randomized control trial of bupivacaine and fentanyl versus fentanyl-only for epidural analgesia during the second stage of labor. Anesthesiology. 2015; 122: 172-177.

[20] Heesen M, Böhmer J, Klöhr S, Hofmann T, Rossaint R, Straube S. The effect of adding a background infusion to patient-controlled epidural labor analgesia on labor, maternal, and neonatal outcomes. Anesthesia \& Analgesia. 2015; 121: 149-158.

[21] Capogna G, Camorcia M, Stirparo S, Farcomeni A. Programmed intermittent epidural bolus versus continuous epidural infusion for labor analgesia: the effects on maternal motor function and labor outcome. A randomized double-blind study in nulliparous women. Anesthesia \& Analgesia. 2011; 113: 826-831.

[22] Feng S, Xu S, Ma L, Li C, Wang X, Yuan H, et al. Regular intermittent bolus provides similar incidence of maternal fever compared with continuous infusion during epidural labor analgesia. Saudi Medical Journal. 2015; 35: 1237-1242.

[23] Leo S, Ocampo CE, Lim Y, Sia AT. A randomized comparison of automated intermittent mandatory boluses with a basal infusion in combination with patient-controlled epidural analgesia for labor and delivery. International Journal of Obstetric Anesthesia. 2010; 19: 357364.

[24] Patkar C, Vora K, Patel H, Shah V, Modi M, Parikh G. A comparison of continuous infusion and intermittent bolus administration of 0.1 ropivacaine with 0.0002 fentanyl for epidural labor analgesia. Journal of Anaesthesiology Clinical Pharmacology. 2015; 31: 234-238.

[25] Sng BL, Zhang Q, Leong WL, Ocampo C, Assam PN, Sia ATH. Incidence and characteristics of breakthrough pain in parturients using computer-integrated patient-controlled epidural analgesia. Journal of Clinical Anesthesia. 2015; 27: 277-284.

[26] Anim-Somuah M, Smyth R, Howell C. Epidural versus non-epidural or no analgesia in labour. The Cochrane Database of Systematic Reviews. 2011; 4: CD000331.

[27] Cossu AP, De Giudici LM, Piras D, Mura P, Scanu M, Cossu M, et al. A systematic review of the effects of adding neostigmine to local anesthetics for neuraxial administration in obstetric anesthesia and analgesia. International Journal of Obstetric Anesthesia. 2015; 24: 237 246.

[28] Hung TH, Hsieh TT, Liu HP. Differential effects of epidural analgesia on modes of delivery and perinatal outcomes between nulliparous and multiparous women: a retrospective cohort study. PLoS ONE. 2015; 10 : e0120907.

[29] El-Kerdawy H, Farouk A. Labor analgesia in preeclampsia: remifentanil patient controlled intravenous analgesia versus epidural analgesia. Middle East Journal of Anaesthesiology. 2010; 20: 539-545.

[30] Ranganathan P, Golfeiz C, Phelps AL, Singh S, Shnol H, Paul N, et al. Chronic headache and backache are long-term sequelae of unintentional dural puncture in the obstetric population. Journal of Clinical Anesthesia. 2016; 27: 201-206.

[31] Wong CA, Scavone BM, Peaceman AM, McCarthy RJ, Sullivan JT, Diaz NT, et al. The risk of cesarean delivery with neuraxial analgesia given early versus late in labor. The New England Journal of Medicine. 2005; 352: 655-665

[32] Wang F, Shen X, Guo X, Peng Y, Gu X. Epidural analgesia in the latent 
phase of labor and the risk of cesarean delivery: a 5-year randomized controlled trial. Obstetric Anesthesia Digest. 2010; 30: 185-187.

[33] Torvaldsen S, Roberts CL, Bell JC, Raynes-Greenow CH. Discontinuation of epidural analgesia late in labour for reducing the adverse delivery outcomes associated with epidural analgesia. The Cochrane Database of Systematic Reviews. 2004; 4: CD004457.

[34] George RB, Allen TK, Habib AS. Intermittent epidural bolus compared with continuous epidural infusions for labor analgesia: a systematic review and meta-analysis. Anesthesia and Analgesia. 2013; 116: 133144.

[35] Butwick AJ. 2012 Gerard W. Ostheimer lecture-what's new in obstetric anesthesia? International Journal of Obstetric Anesthesia. 2012; 21: 348356.

[36] Camorcia M, Capogna G, Stirparo S, Berritta C, Blouin JL, Landau R. Effect of $\mu$-opioid receptor A118G polymorphism on the ED50 of epidural sufentanil for labor analgesia. International Journal of Obstetric
Anesthesia. 2012; 21: 40-44.

[37] Pettersson FD, Grönbladh A, Nyberg F, Sundström-Poromaa I, Åkerud H. The A118G single-nucleotide polymorphism of human $\mu$-opioid receptor gene and use of labor analgesia. Reproductive Sciences. 2012; 19: 962967.

[38] Zhang N, Xu M. Effects of epidural neostigmine and clonidine in labor analgesia: a systematic review and meta-analysis. Journal of Obstetrics and Gynaecology Research. 2015; 41: 214-221.

How to cite this article: Xue Bai, Zi-Xuan Song, Yu-Xiao Wan, Zhi-Qiang Feng, Dong-Hai Yu, Dan-Dan Zhang. Mapping theme trends and knowledge structure of labor analgesia: a quantitative, co-word biclustering analysis of data in 2000-2020. Signa Vitae. 2021;17(4):98-107. doi:10.22514/sv.2021.012. 